\title{
Electron tomography of chemotaxis receptor signaling complex
}

\author{
Xin Meng*, Gongpu Zhao*, Qilong Mao*, Xiongwu Wu**, and Peijun Zhang* \\ * Department of Structural Biology, University of Pittsburgh, Pittsburgh, PA 15260 \\ ** Laboratory of Computational Biology, National Heart, Lung and Blood Institute, NIH, \\ Bethesda, MD 20892
}

The movement of bacteria in response to external stimuli represents a paradigm of broad general interest for the understanding of mechanisms underlying signal transduction across cell membranes. Bacterial chemoreceptors respond to changes in concentration of extracellular ligands by undergoing conformational changes of a signaling complex consisting of transmembrane chemotaxis receptors, the histidine autokinase CheA and the coupling protein CheW, which initiate a series of signaling events, leading ultimately to regulation of flagellar motor rotation [1]. Moreover, a few thousand receptor signaling complexes form higher order assemblies at the cell pole and these are responsible for the extraordinary gain in chemotaxis signaling [2]. Despite the determination of high resolution structures of the individual soluble domains of the receptor, CheA, and CheW, the structure of the receptor-CheA-CheW ternary complex and the architecture of the extended higher order assembly have remained elusive. The large size and dynamic nature make the signaling complex a difficult target for any structural study. Here, we have used electron tomography combined with 3D averaging to determine the 3D structure of the signaling complex that assembles into crystalline 2D arrays on lipid monolayers (Fig.1). The 3D density map of the ternary complex averaged with 2100 hexameric repeating units from raw 3D tomograms of negatively stained arrays displays pseudo- 6 fold related trimers of receptor dimer molecules (Fig 1b-d), and pseudo-2 fold structure at the CheA and CheW region (Fig 1e, Fig. 2d). The receptor dimer molecules within the trimer unit are fully resolved and a receptor trimer of dimer model can be fitted into the densities well (Fig. 2b).

We have further employed a 3D classification strategy described in our previous studies [3] to separate different conformations present in the signaling complex. We found two major classes that display moderate differences at the CheA and CheW regions (Fig. 2f-h), whereas the receptor cytoplasmic regions are essentially the same (Fig. 2e). These two classes may represent two different states of the ternary complex, particularly with changes at CheA and CheW molecules, suggesting important conformational roles of the signaling molecules in mediating signal transduction in bacterial chemotaxis. It is also possible that observed differences might arise from the staining artifact. Structural analysis of frozen-hydrated monolayer using cryoET and higher resolution structures from 2D monolayer crystals will further allow reliable fitting of the molecular models into density maps. These analyses will shed new light on the molecular mechanism of signaling and signal processing in bacterial chemotaxis.

\section{References}

[1] G. L. Hazelbauer et al. (2008). Trends Biochem Sci 33, 9-19.

[2] P. Zhang et al., (2007). Proc Natl Acad Sci U S A 104, 3777-81.

[3] C. M. Khursigara et al. (2008). Proc Natl Acad Sci U S A 105(43):16555-60. 
Figure 1. Electron-tomography of negatively stained TarCF/CheA/CheW complex assembled on lipid monolayer. (a) A projection images of reconstituted signalingcomplex with His 6 TarCF, Che A and CheW onto tipid monolayer Hexagonal micro-crstalline arrays of the complex are formed. Insert, Fourier transform of crystalline area indicates antit cell dimension of $\tau 102 \mathrm{~A}$. (b-e) Slices of 3D density map of the signaling complex assembly unit by electron-tomography and $3 \mathrm{D}$ averaging. b\&c, receptor region (b\&c), d\&e, Che A/CheW region.
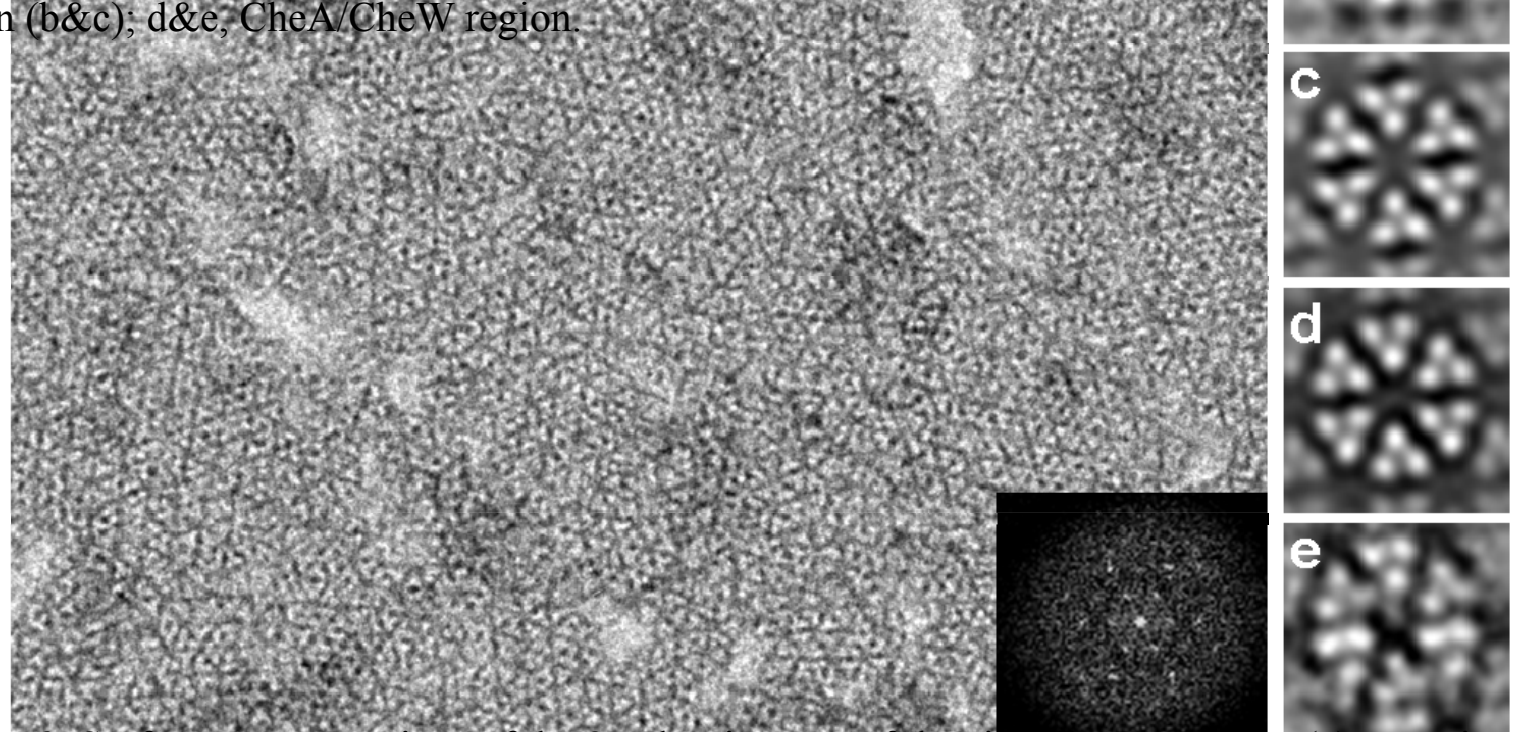

Figure 2. Surface representations of the $3 B$ density map of the signamng cumpru. (a) Top view and (b) cut-away view with trimer of the dimer TarCF docked. TarCF is in gold and CheA and CheW are in blue. (c) Top view of the receptor region shows a pseudo-6 fold symmetry. (d) Bottom view of CheA/CheW region shows a pseudo- 2 fold symmetry. One asymmetric unit in the lattice is marked with oval. (e-h) 3D classification of the TarCF/CheA/CheW complexes. Two major classes are shown as surface representation in gold and blue, respectively. While the receptor regions are similar in two classes (a), the CheA and CheW regions are different (f-h). ( $g$ \& h) Bottom views of the

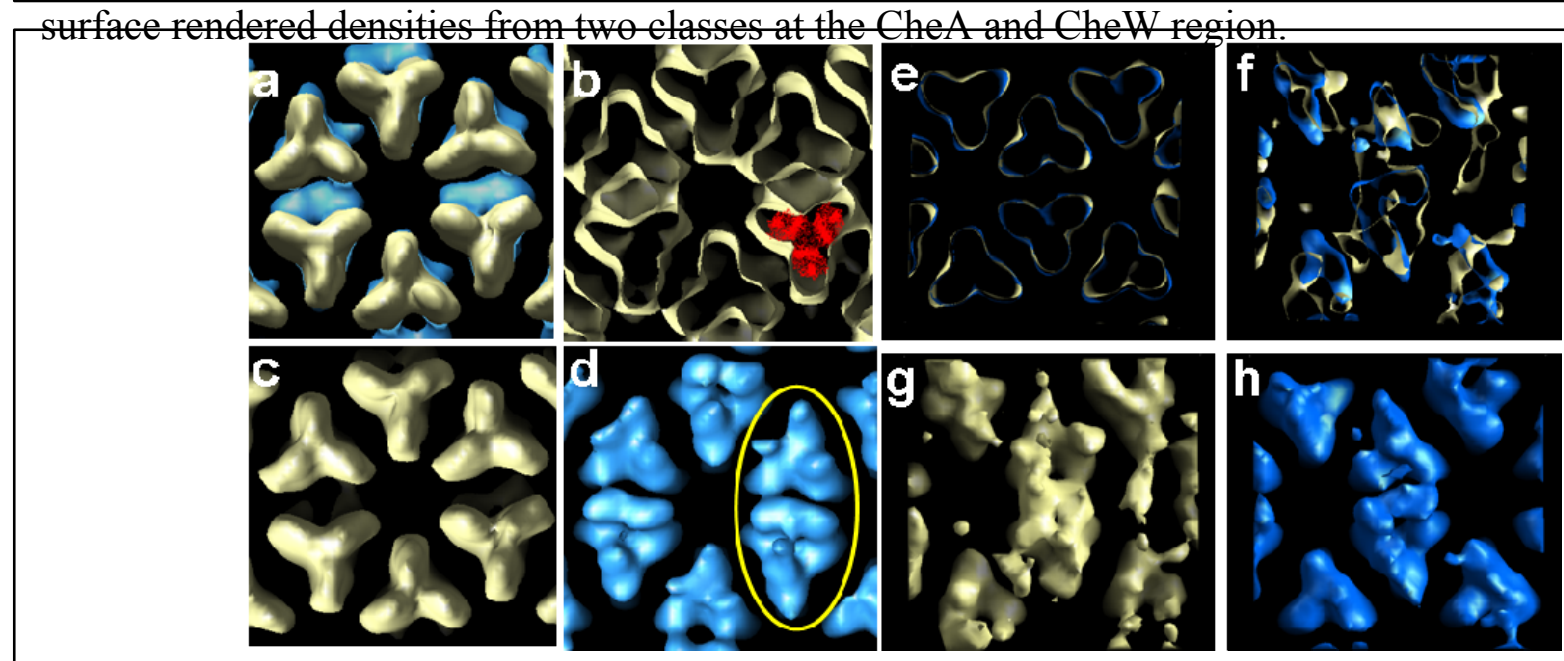

